\title{
Technische Bedingungen in der historischen Entwicklung der Meteorologie
}

\author{
Von Emil J. Walter, Zürich
}

Ein besonders wichtiges Problem der allgemeinen Wissenschaftsgeschichte bilden die technischen Voraussetzungen der wissenschaftlichen Forschung. Seit die Naturwissenschaften von reiner Beobachtung und Beschreibung zur apparatorientierten experimentellen Methode übergegangen sind, kann die Geschichte einzelner Fachwissenschaften ohne eingehende Berücksichtigung der verwendeten Apparate und experimentellen Arbeitsmethoden nicht verstanden werden. Und Apparate, Instrumente und Arbeitsmethoden sind ihrerseits weitgehend abhängig vom allgemeinen Stande der handwerklichen und industriellen Technik. Die entscheidende Abhängigkeit der Meteorologie und wissenschaftlichen Wetterprognose von Stand und Ausbau des Nachrichtenwesens bildet ein besonders schönes und eindrückliches Beispiel für die Abhängigkeit der wissenschaftlichen Forschung von ihren technischen Grundlagen.

Die Meteorologie ist sowohl eine physikalische als auch eine geographische Wissenschaft. Als Physik der Atmosphäre ist sie auf die Messung physikalischer Zustandsgrößen angewiesen. Als geographische Wissenschaft bedarf die Meteorologie der gleichzeitigen Messung dieser Zustandsgrößen auf einem ausgedehnten Gebiete der Erdoberfläche. Erfindung entsprechender Instrumente (Barometer, Thermometer, Hygrometer usw.) bilden die eine, Erfindung von Methoden der Nachrichtenübermittlung (Telegraph usw.) die zweite Voraussetzung der Entwicklung der Meteorologie.

Vor der Erfindung des Barometers ist die Meteorologie entweder eine Beschreibung klimatischer Verhältnisse nach dem Vorbild des Aristoteles oder Volksmeteorologie. Astrometeorologische Kalender, Wetterbüchlein, «Purenkalender» und «Hundertjähriger Kalender» belegen ein mächtiges soziales Bedürfnis, wie es kurz nach der Erfindung des Buchdruckes in einer Art Inflation meteorologischer Schriften zum Ausdruck kam. Das Bedürfnis nach mehr oder weniger sicheren Wetterregeln und Wetterzeichen reichte aber nicht aus zur Schaffung einer besonderen Fachdisziplin. Erst durch die Erfindung der entsprechenden Meßinstrumente wurde die eine instrumentelle Voraussetzung für die objektive Beobachtung der Atmosphäre an einzelnen Orten geschaffen. Damit wurde die erste Stufe der wissenschaftlichen Meteorologie möglich. In diese Zeitperiode von der Mitte des 17. bis zur Mitte des 19. Jahrhunderts fallen die ersten Versuche zur Schaffung von einzelnen Wetterbeobachtungsnetzen, die wichtige Vorstudien möglich machten. Die zweite Stufe der wissenschaftlichen Meteorologie setzte die Ausbildung einer leistungsfähigen Nachrichtentechnik voraus, 
denn nur in diesem Falle konnte zur Zeichnung von täglichen Wetterkarten übergegangen werden.

Die Meteorologie ist eine geographische Wissenschaft eigener Art. Der Geograph, beschäftige er sich mit Länderkunde, Orographie, physischer Geographie, Tier- und Pflanzengeographie oder Kultur- und Wirtschaftsgeographie, hat es mit relativ stationären Verhältnissen zu tun. Die Dimension der Zeit greift in die Betrachtungsweise des Geographen in verhältnismäßig leicht überschaubarer Art und Weise ein. Die Witterungserscheinungen, das wissenschaftliche Objekt der Meteorologie, erstrecken sich über große Entfernungen und unterliegen einem steten Wechsel. Die Meteorologie ist eine dynamische Wissenschaft.

Sobald die Nachrichtentechnik genügend entwickelt war, wurde die Meteorologie vor allem zu einer Frage der Organisation und Koordination der Beobachtungsnetze. Die Beobachtungstermine der einzelnen Stationen mußten sorgfältig aufeinander abgestimmt werden. Das Abkommen über eine großzügige, europäische und amerikanische Stationen zusammenschließende simultane Beobachtung ist auf Vorschlag des amerikanischen Generals A.J.MYer schon im Jahre 1874 abgeschlossen worden.

Die wichtigsten Abschnitte der geschichtlichen Entwicklung der Meteorologie sind:

I. Vorinstrumentelle Meteorologie (von Aristoteles bis Torricelli; Mythologische Meteorologie, Volksmeteorologie)

a) Griechische «Meteorologie» (Hippokrates um 400 v. Chr.; Aristoteles 384-322 v. Chr.; Theophrast 373-286 v. Chr.)

b) Astrometeorologische und andere Prognostiken (Hundertjähriger Kalender) nach der Erfindung des Buchdrucks vom 16. bis zum 18. Jahrhundert. Sammlung von bäuerlichen Wetterregeln und Wetterzeichen.

c) Rein deskriptive lokale Meteorologie in Form von Wetterchroniken.

II. Instrumentelle Meteorologie (von 1645 bis 1830)

a) 1643 Erfindung des Barometers durch Torricelli.

b) Sporadischer Beginn langjähriger systematischer lokaler Wetterbeobachtungen unter Verwendung von Barometer, Thermometer, Windfahne, Hygrometer und andern Instrumenten im 17. und 18. Jahrhundert.

c) Schaffung von einzelnen Wetterbeobachtungsnetzen (z. B. 1654/1667 durch die florentinische «Accademia del Cimento»; 1717/1726 «Breslauer Sammlung»; 1781/1792 Meteorologische Beobachtungen der «Mannheimer Societät» mit 39 internationalen Beobachtungsstationen).

III. Synoptische und dynamische Meteorologie (von 1830 bis zur Gegenwart)

a) Ausbau der nationalen Wetterbeobachtungsdienste zwischen 1800 und 1850 aus zum Teil spontan entstandenen Wetterstationen. 


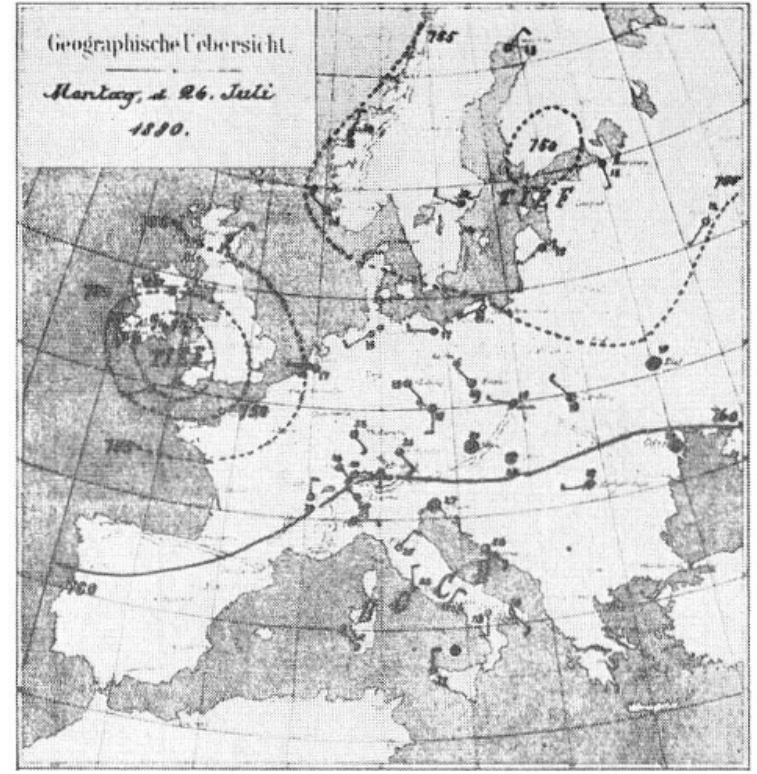

Abb. 1

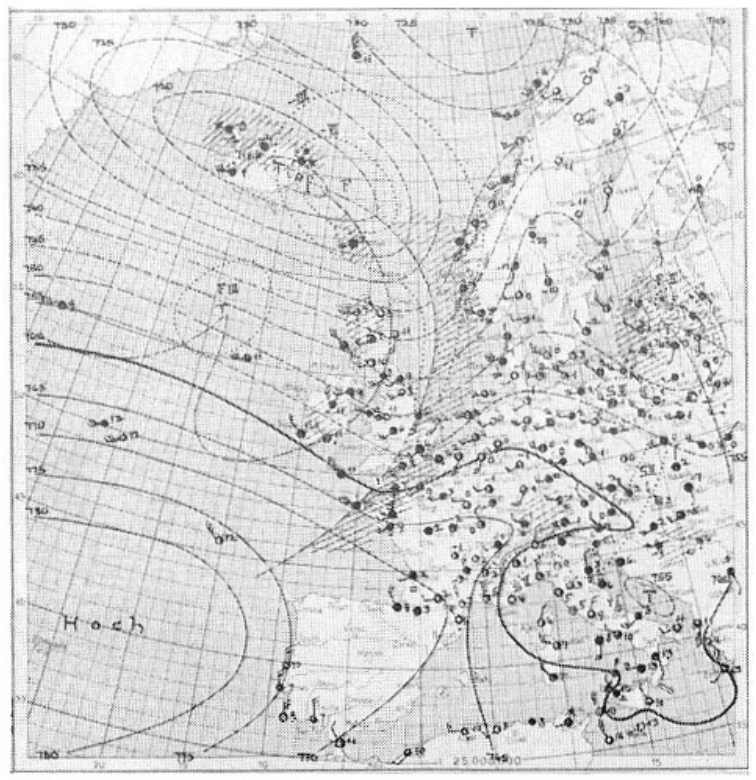

Abb. 3

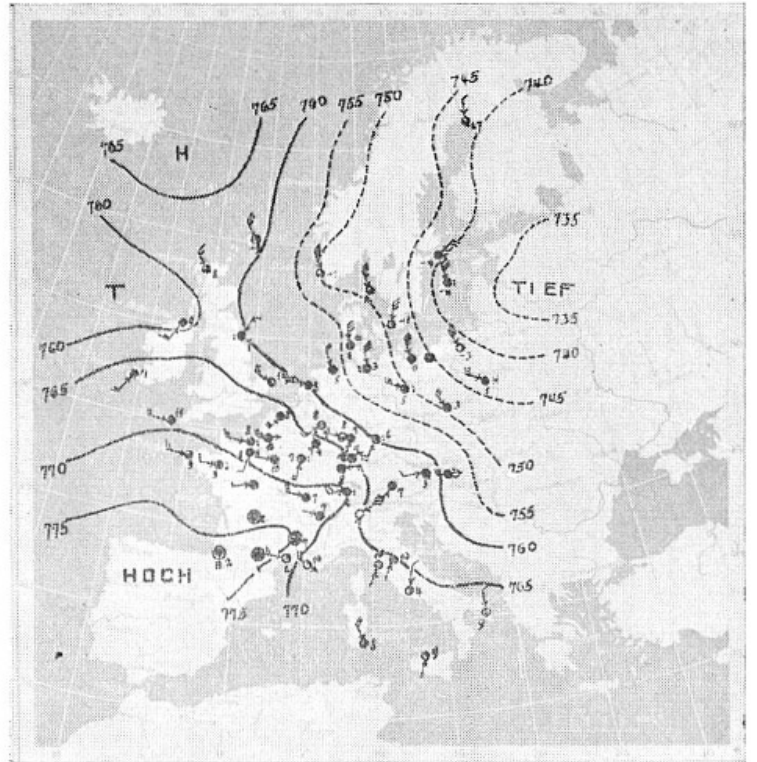

Abb. 2

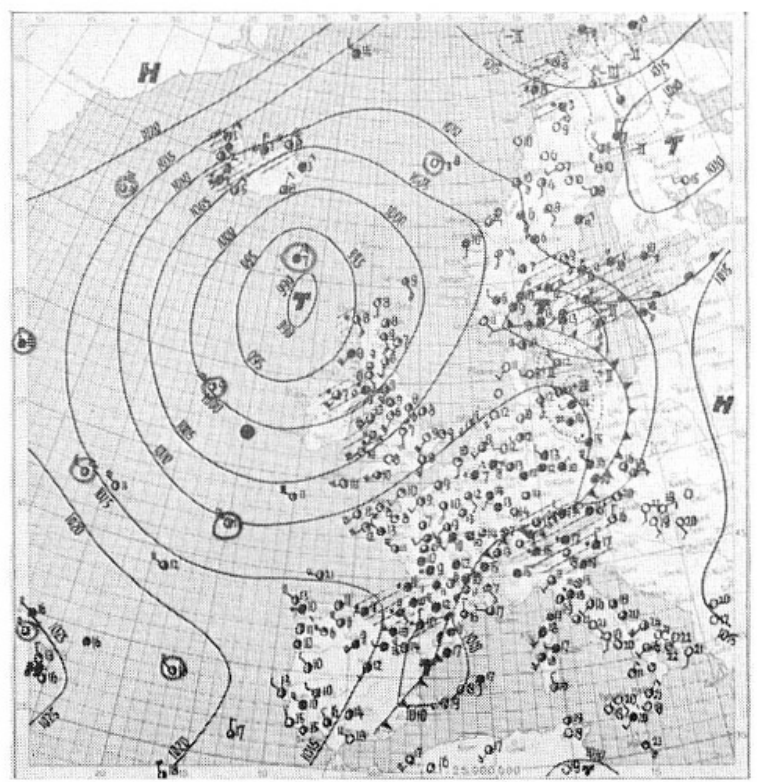

Abb. 4

Abb. 1. Wetterkarte vom 26. Juli 1880. Die Zahl der Beobachtungsstationen ist sehr klein. Aus einzelnen Ländern sind überhaupt keine Meldungen eingegangen

Abb. 2. Wetterkarte vom 24. Januar 1921. Das Beobachtungsfeld erstreckt sich nur auf Westeuropa Abb. 3. Wetterkarte vom 28. Januar 1938. Das Beobachtungsnetz ist stark erweitert worden. Die Lehre von den Wetterfronten kommt in der Karte zum Ausdruck

Abb. 4. Wetterkarte vom 30. Mai 1949. Das Beobachtungsfeld erstreckt sich von Osteuropa bis nach den USA. Auf dem Atlantischen Ozean sind Wetterschiffe (durch Kreise hervorgehoben) stationiert 
b) Einführung der Wetterkarte parallel zur Entwicklung des Telegraphendienstes (1850/65). [Erste Zeichnung von synoptischen Karten durch H.W.Brandes (1820), E. Loomis (1836), Espy (1841). 1837 Morseschreibtelegraph. 1851 Synoptische Karten an der Londoner Weltausstellung. 1863 «Bulletin International» des französischen Wetterdienstes mit synoptischen Karten.]

c) Ausbau und Internationalisierung der synoptischen Methode (1865/1900). 1. Durch sorgfältige Analyse von täglichen synoptischen Karten werden die Grundlagen für eine wissenschaftliche Wetterprognose geschaffen. Die nationalen Wetterdienste organisieren ihre internationale $\mathrm{Zu}$ sammenarbeit und beginnen internationale synoptische Karten "zu veröffentlichen (1865/1880).

2. Langsamer Ausbau der verschiedenen landmeteorologischen Dienste 1880/1900. Theoretische Stagnation.

d) Zyklonen- und Polarfronttheorie und Luftmassenlehre (1900/1925).

Die norwegische Schule unter BJERKnes entwickelt die moderne Zyklonen- und Polarfronttheorie um das Jahr 1910. Sie wird in den zwanziger Jahren in Verbindung mit der Luftmassenlehre allgemein anerkannt. Durch die Einführung des Radio können auch Wettermeldungen von Seeschiffen täglich in die Wetterkarten eingetragen werden.

e) Erforschung der mittleren und oberen Troposphäre und Ausbau der Flugmeteorologie (1925/1950).

Das Netz der Beobachtungsstationen wird immer dichter und geographisch immer weiter ausgedehnt (auf dem Ozean nach dem Zweiten Weltkrieg Wetterschiffe; Ausbau des arktischen Beobachtungsnetzes).

Die Radiotechnik erlaubt die Durchgabe der Beobachtungen von zahlreichen Beobachtungsterminen.

Die Flugmeteorologie wendet sich der systematischen Erforschung der mittleren und oberen Schichten der Troposphäre durch Wetterflüge, Pilotballone und Radiosonden zu.

Aufschwung der theoretischen Meteorologie: Ausbau der dynamischen Meteorologie, Eiskeimtheorie des Regens von Bergeron, Theorie der Gewitterelektrizität von FindeISEN.

Im Anhang I stellten wir eine chronologische Übersicht über die Entwicklung der Meteorologie zusammen, der Anhang II enthält die entsprechenden chronologischen Angaben über die Entwicklung der Nachrichtentechnik. Anhand dieser Übersichten sei es uns gestattet, auf einige wichtige Entwicklungsphasen aufmerksam zu machen. Während der Periode der instrumentellen Meteorologie, die den Übergang von der rein lokalen und mehr oder weniger zufälligen Beobachtung zur Beobachtung mit Beobachtungsnetzen bringt, wird die BEAUfort-Skala der Windstärken und die Klassifikation der Wolkenformen geschaffen. H.W.Brandes wertet um 1820 die Beobachtungen des Beobachtungsnetzes der 
Mannheimer Societät erstmals zur Zeichnung von synoptischen Wetterkarten aus. Mit der Schaffung von amtlichen Wetterdiensten werden meteorologische Theorien formuliert, so das Gesetz der Stürme von Dove, die Regel von BuYs-Ballot über die Richtung der Windströmungen, die Föhntheorien von Dove, WiLD und HaNN und zu Beginn des 20. Jahrhunderts die Wellentheorie der Zyklonen, die Polarfronttheorie und die Luftmassenlehre vor allem von der norwegischen Schule unter V. und J. BJERKNEs.

Die Aufgabe der amtlichen Meteorologie wird zunächst als Wetterprognose im Dienste der Landwirtschaft und der Touristik gesehen. Mit der Ausbreitung des Flugverkehrs hat die Bedeutung der Meteorologie stark zugenommen, werden immer größere öffentliche Mittel dafür aufgewendet, wird das Beobachtungsnetz verdichtet und erweitert, wie sich aus der Wiedergabe von schweizerischen Wetterkarten aus den Jahren 1880, 1921, 1938 und 1949 ergibt (siehe Abb. 1-4). Trotz den bedeutsamen Fortschritten der meteorologischen Forschung, der Ausdehnung des Beobachtungsfeldes auch in lotrechter Richtung oder durch die Errichtung von Beobachtungsstationen in der Arktis und auf Wetterschiffen auf dem Ozean bestehen für die wissenschaftliche Prognose grundsätzlich gewisse Grenzen: Die meteorologischen Vorgänge verfügen über keine festen Randbedingungen, welche erlauben würden, mit Sicherheit, nicht nur mit einer gewissen, wenn auch oft recht hohen Wahrscheinlichkeit, zu prognostizieren.

Zusammenfassend darf aus der Entwicklung der Meteorologie wohl der Schluß gezogen werden, daß die wissenschaftliche Forschung von vier Bedingungsgruppen abhängig ist :

1. Vom Bedürfnis nach bestimmten wissenschaftlichen Erkenntnissen. Das Bedürfnis nach Wetterprognosen und Erklärung der Wettererscheinungen ist so alt wie der Ackerbau.

2. Vom Stande der Technik. Wissenschaftliche Meteorologie wurde erst möglich nach Erfindung entsprechender Meßinstrumente und Nachrichtenmittel.

3. Von den experimentellen und wirtschaftlichen Möglichkeiten der Forschung des betreffenden Fachgebietes. Die Verdichtung der Beobachtungsnetze der Meteorologie steht in engstem Zusammenhang mit der Entwicklung des Flugwesens und der damit zusammenhängenden Bereitschaft, größere finanzielle Mittel für den Wetterdienst im allgemeinen und den Flugwetterdienst im besonderen aufzuwenden.

4. Von der Persönlichkeit des wissenschaftlichen Forschers und seinem Erkenntniswillen.

\section{Anhang I. Chronologische Übersicht der Entwicklung der Meteorologie}

460(?)-375(?) v. Chr.: Hippokrates, griechischer Arzt von Kos, behandelt den Einfluß des Ortes und der Luft auf die menschliche Gesundheit. - Regenmessungen in Indien.

384-322 v. Chr.: Aristoteles. Von den 42 Kapiteln der vier Bücher der «Meteorologie» behandeln 15 Kapitel die Wissenschaft der Atmosphäre (wesentliche Punkte: 
Das Warme hat die Tendenz zu steigen. Klassifikation der Winde. Beschreibung von Tau und Reif u. ä.). Hauptwerk und Vorbild bis zum 18. Jahrhundert.

373-286 v. Chr.: Theophrast von Lesbos, bedeutendster Schüler des Aristoteles. Fragmente über Wetterzeichen (80 Regenzeichen, 45 Windzeichen, 15 Sturmzeichen, 24. Zeichen für schönes Wetter, 7 Zeichen für die kommende Witterung eines Jahres).

100-200 n. Chr. Regenmessungen in Palästina.

1442 Regenmessungen in Korea unter König SEJo.

1508 Bauernpraktik (Büchlein mit bäuerlichen Wetterregeln; mindestens vierzig Auflagen).

1470-1600 Blütezeit der astrometeorologischen Prognostiken und Wetterbüchlein mit Wetterregeln und Wetterzeichen (z. B. 1524 J.Stöfflens Prognose für das Jahr 1524; 1553 Purenkalender im Zürcher Verlag Froschauer).

Um 1612 Einführung des Thermometers durch den Holländer C. Drebbel (1608) und den Italiener Santorio. Die Bezeichnung des Thermometers kommt zwischen 1620 und 1624 auf. SANTORIO gebraucht das Thermometer als erster zur Messung des Fiebers. Das Thermoskop, die Vorstufe des Thermometers, ist von Heron (130 n. Chr.) und Philon (170 n. Chr.) erfunden worden.

1643 Viviani führt auf Anregung E. Torricellis (1608-1647) den bekannten Quecksilberversuch durch. Dieses «Experience d'Italie» wird erst 1646 in Rouen durch Pater Mersenno (1588-1648) und Pascal wiederholt, weil zunächst keine Glasröhren aufzutreiben waren.

19. September 1648: PÉrIer, der Schwager von Blaise Pascal (1623-1662) führt auf dessen Vorschlag den Torricellischen Versuch auf dem Puy-de-Dôme bei Clermont-Ferrand durch. Entsprechend dem Höhenunterschied von rund 970 Metern zeigt sich ein Unterschied der Länge der Quecksilbersäule von 3 Zoll $11 / 2$ Linien $(=79,4 \mathrm{~mm})$. Pascal scheint die Anregung zu diesem wichtigen Experiment von Descartes empfangen zu haben.

1649/51 Erste korrespondierende Barometerbeobachtungen in Clermont-Ferrand, Paris und Stockholm.

1654/1667 Meteorologisches Beobachtungsnetz der durch Ferdinand II. gegründeten florentinischen «Accademia del Cimento» (erst vier, dann sieben italienische und vier ausländische Stationen). Die Akademie stellte auch Weingeist- und Quecksilberthermometer her.

1662 R. BoyLe (1627-1691) veröffentlicht das Gesetz über den Zusammenhang von Druck und Volumen bei Gasen.

1666/1667 R. Hooke (1635-1703) stellt ein Schema für meteorologische Beobachtungen auf ( $A$ Scheme at one view representing to the Eye the Observations of the Weather for a Month: Angaben über Datum, Sonnenstand, Mondviertel, Windrichtung und Windstärke, Grade der Wärme und Kälte, der Trockenheit und Feuchtigkeit, Bewölkung, besondere Erscheinungen und besondere Bemerkungen)

Um 1670 Allgemeine Verwendung der ToRricelLischen Röhre als «Wetterglas».

1676 MARIOTTE (1620[ ?]-1684) führt den Ausdruck Barometer (Essai sur la nature de l'air) ein und präzisiert das Boylesche Gasgesetz. J.C.STurm, Professor in 
Altdorf, Lehrer von J.J.Scheuchzer, gibt das Collegium experimentale, eine der ältesten meteorologischen Instrumentenkunden, heraus.

1688 E. Halley (1656-1742) veröffentlicht eine erste Weltkarte der Passat- und Monsunwinde.

1700 Erste Ausgabe des sogenannten Hundertjährigen Kalenders durch den Arzt Christoph Hellwig (1663-1721) auf Grund der Wetterbeobachtungen des Cisterzienser Abtes vom Kloster Langheim bei Lichtenfels Moritz KNAUER (1613-1664): Partikularwitterung 1650/54 (Calendarium æeconomicum et perpetuum). Nachkontrolle ergab im 19. Jahrhundert 39\% Treffer, $44 \%$ Falschprognosen, 17\% unentschiedene Fälle. G. Hellmans hat 159 Ausgaben des Hundertjährigen Kalenders nachgewiesen.

1713/1715 FaHRENHEIT (1686-1735) konstruiert Quecksilber- und Weingeistthermometer in der heute üblichen Form, wahrscheinlich auf Grund einer 1709 von OLAus Römer (1644-1710) empfangenen Anregung: $0^{\circ}$ Kältemischung, $32^{\circ}$ Schmelzpunkt des Eises, $96^{\circ}$ Blutwärme.

1717/1726 Breslauer Sammlung von Meteorologischen Beobachtungen (J.J.SchEUchzER, Berichterstatter für die Stationen Zürich und St. Gotthard).

1730 Ausführliche Studie RÉAumurs (1683-1757) über Thermometrie. Schlägt eine Skala von $1000^{\circ}$ für schmelzendes Eis und $1080^{\circ}$ für siedendes Wasser vor.

1735 G.HADLEY (1685-1744) berücksichtigt für die Erklärung der Abweichung der Passatwinde von der Nord-Süd-Richtung die Erdrotation.

1742 Der Schwede Celsius (1701-1744) führt die hundertteilige Thermometerskala ein: $0^{\circ}$ für siedendes Wasser, $100^{\circ}$ für schmelzendes Eis. Sein Schüler Strömer vertauscht die beiden Fixpunkte.

1752 Benjamin Frankin (1706-1790) beweist durch Drachenaufstiege die elektrische Natur des Blitzes und erfindet den Blitzableiter.

1774. Traité de météorologie von Pater Louss CotTe (1740-1815), eines der ersten modernen, sich vom aristotelischen Vorbild befreienden Lehrbücher der Meteorologie (1776-1786 systematische meteorologische Beobachtungen in Paris).

1780 LavoIsIER (1743-1794) schlägt in Verbindung mit LAMARCK (1744-1829) die Errichtung eines meteorologischen Beobachtungsnetzes vor.

1781-1792 Meteorologische Beobachtungen der Mannheimer Societät. Erstes systematisch aufgebautes internationales Beobachtungsnetz. Gegründet durch den Kurfürsten KarL Theodor von der Pfalz, geleitet durch den geistlichen Rat J.J.Hemmer (1733-1790). 39 Beobachtungsstationen (davon 14 deutsche), die mit den gleichen Instrumenten ausgestattet wurden und deren Beobachter auf Grund genauer Instruktionen zu gleichen Beobachtungsterminen (7 Uhr, $2 \mathrm{Uhr}$ nachmittags, $9 \mathrm{Uhr}$ ) die Beobachtungen in 13 Rubriken eintrugen. Umrechnung der Beobachtungen in Mannheim und Veröffentlichung als Ephemerides societas meteorologica Palatina in den Jahren 1785 bis 1794.

1782 Six erfindet das nach ihm benannte Minimum- und Maximumthermometer (von ihm fälschlich als «Thermometrograph» bezeichnet).

1784. Erster meteorologischer Ballonaufstieg von Dr. JoHN JEFFrIES und BLANCHARD.

1787 H.B. DE SAUSSURe (1740-1799) besteigt den Montblanc. SAUSSURE beschäftigt sich eingehend mit Hygrometrie. 
1795 Roмme schlägt die Übermittlung von Wettermeldungen durch den optischen Telegraphen der Gebrüder Chappe vor.

1800 John Datton (1766-1844), Meteorologe und Begründer der chemischen Atomtheorie, veröffentlicht wichtige Untersuchungen über die Kondensation des Wasserdampfes.

1802 GAY LusSaC (1778-1850) zeigt, daß sich alle Gase beim Erwärmen mit dem gleichen Ausdehnungskoeffizienten ausdehnen. Der Ausdehnungskoeffizient selbst wurde schon von LAMBERT (1728-1777) bestimmt und posthum veröffentlicht (1779).

1803 On the Modifications of Clouds von LUKE Howard (1778-1864). Erste wissenschaftliche Klassifikation der Wolken.

1806 Sir Francis Beaufort (1774-1857) stellt die nach ihm benannte Skala der Windstärken auf.

1814 Charles Wells (1757-1817) formuliert eine Theorie des Taus, welche die Entstehung des Taus durch die nächtliche Ausstrahlung von Wärme erklärt.

1817 Alexander von Humboldt (1769-1859) publiziert in den Memoirs de la Société d'Arcueil anhand von Angaben von lediglich sechzig Stationen Karten über die Verteilung der Temperatur und des Luftdruckes auf der Erde (Isothermen, Isobaren).

1820 Beiträge zur Witterungskunde von H.W.Brandes (1777-1834) (synoptische Karten nach den Beobachtungen der Soc. Palatinae und L. CotTE vom Jahre 1783. Die Karten sind verloren gegangen. Die Karte vom 6. März 1783 ist die erste synoptische Karte mit Isobaren und eingezeichneten Windrichtungen).

1823 Pictex schlägt der Schweizerischen Naturforschenden Gesellschaft vor, ein meteorologisches Beobachtungsnetz von zwölf Stationen zu schaffen. Der Versuch wird im Jahre 1837 wieder aufgegeben.

1826 Dissertatio physica de repentinis variationibus in pressione atmosphaerae observatis von H.W.Brandes (Studie über synoptische Karten von zwei Depressionen vom 24. bis 26 . Dezember 1821 und 2. bis 3 . Februar 1823).

1828 L.A. J. QUETELET (1796-1874) beginnt regelmäßige Beobachtungen über periodische meteorologische Erscheinungen und fördert speziell die Kenntnis vom Beginn der Pflanzenblüte.

1820/1850 Ausbau der nationalen Beobachtungsnetze. Z. B. bestanden in Deutschland nach G. HeLlmaNN 1701/10 6 Stationen; 1761/70 15; 1771/80 38; 1781/90 67; $1791 / 180035 ; 1801 / 1020 ; 1811 / 2053 ; 1821 / 30143 ; 1831 / 40161$ und 1841/50 169 Stationen.

1831/36 Lehrbuch der Meteorologie von Ludw. Fr. Käмtz (1801-1867).

1836/42 ELIAS Loomis (1811-1889) veröffentlicht Studien über die Stürme, schlägt die Konstruktion von halbtäglichen meteorologischen Karten in den Vereinigten Staaten vor.

1838 Monse meldet ein Patent für einen Schreibtelegraphen an.

1841 Philosophy of Storms von J. P. EsPY (1785-1860). Organisiert simultane tägliche Wetterbeobachtungen, sammelt synoptische Karten von über 1100 Tagen. Wird 1843 Chef des amerikanischen «Meteorological Bureau of the War Department». 
KARL Kreil (1798-1862), Assistent der Prager Sternwarte, nach 1851 Direktor der Zentralanstalt für Meteorologie und Erdmagnetismus in Wien, schlägt die telegraphische Übermittlung von Wetterbeobachtungen vor.

Julius Robert Mayer (1814-1878) formuliert das Prinzip der Erhaltung der lebendigen Kraft als allgemeines Naturgesetz und berechnet aus der Differenz der spezifischen Wärme der Gase bei konstantem Druck und konstantem Volumen das Wärmeäquivalent.

1848 JoHN BaLL unterbreitet der British Association einen detaillierten Vorschlag zur telegraphischen Übermittlung von Wetternachrichten.

1849 Henry, Sekretär der amerikanischen Smithsonian Institution macht den Vorschlag, es solle dem Signal Service von jeder Telegraphenstation täglich ein Wetterbericht telegraphiert werden. Vom 14. Juni 1849 ab veröffentlicht James Glaisher in den Daily News regelmäßige Wetterberichte.

1850 James GLAISHER gründet die britische «Meteorological Society».

1851 An der Londoner Weltausstellung werden auf Veranlassung der Postverwaltung - um die Leistungsfähigkeit des telegraphischen Dienstes zu unterstreichen vom 8. August bis zum 11. Oktober täglich synoptische Wetterkarten gedruckt.

1853 Der Amerikaner M.F.MaUrys (1806-1873) veranlaßt die Einberufung der ersten internationalen Konferenz für maritime Meteorologie in Brüssel. Sein Hauptwerk The Physical Geography of the Sea (1855) trägt viel zur Abkürzung der Fahrten der Segelschiffe auf Grund von gründlichen Windstudien bei (z. B. New York-Rio de Janeiro 20 statt 41 Tage; England-Australien 130 statt 250 Tage usw.)

14. November 1854: Ein starker Sturm bringt vor Sebastopol das französische Linienschiff «Henry» zum Kentern und schädigt schwer die alliierten Flotten und Truppen. Von der französischen Regierung wird U. J. J. LE VerRIER (1811-1877; errechnete aus Störungen der Bewegung des Planeten Uranus die Existenz des Neptun) beauftragt, die Ursachen dieser Katastrophe zu untersuchen. Er fordert den Ausbau des französischen Wetterdienstes.

1855/56 Ausbau des französischen Wetterbeobachtungsnetzes und Veröffentlichung von täglichen Witterungsbulletins.

1857 Das Gesetz der Stürme von H.W.Dove (1803-1879). Dove formuliert das Drehungsgesetz des Windes.

$1858 \quad$ Einführung telegraphischer Wetterbulletins in den Vereinigten Staaten.

1860 Am 21. Mai 1860 wird in Holland unter BuYs BaLlot (1817-1890; Entdecker des barischen Windgesetzes) der Sturmwarnungsdienst für die Schiffahrt eingeführt (England 1861, Frankreich 1863, Österreich 1866 usw.)

Vom 3. September 1860 veröffentlicht R. Fitz Roy in der englischen Presse auf Grund von telegraphischen Wettermeldungen tägliche Wetterprognosen.

Auf Vorschlag von Bundesrat Pioda setzt die Versammlung der Schweizerischen Naturforschenden Gesellschaft in Lugano eine Kommission von drei Mitgliedern (Mousson, Wild und Kopp) ein, um in der Schweiz ein meteorologisches Beobachtungsnetz zu organisieren.

1861 Die Versammlung der Schweizerischen Naturforschenden Gesellschaft in Lau- 
sanne stimmt den Anträgen der Meteorologischen Kommission zu und beschließt ein Subventionsgesuch an den Bund und an die Kantone.

Francis Galton versucht in England einen täglichen Wetterbeobachtungsdienst aufzuziehen.

Die Station Mauritius gibt für die Monate Januar bis März synoptische Karten des Indischen Ozeans heraus.

1862 Am 4. Februar beschließt die Schweizerische Bundesversammlung eine Subvention des meteorologischen Dienstes von jährlich 8000 Franken, die 1864 auf 10000,1865 auf 11000,1874 bis 1880 auf 15000 Franken erhöht wird.

1863 Unter Leitung von MARIÉ-Davy veröffentlicht ab 11. September $1863 \mathrm{der}$ französische Wetterdienst ein «Bulletin International» mit synoptischen Karten mit Isobaren.

An der Tagung der Schweizerischen Naturforschenden Gesellschaft in Samedan wird mitgeteilt, daß das schweizerische Beobachtungsnetz über achtzig Stationen umfasse. Der regelmäßige Beobachtungsdienst begann im Dezember 1863. Die Meteorologische Zentralanstalt wurde in der Eidgenössischen Sternwarte in Zürich unter der Leitung von Prof. Rud. WoLF installiert.

1865 Nach dem Tode R.Frtz Roys stellt die Royal Society die Veröffentlichung von Wetterprognosen und Sturmwarnungen ein und bestellt eine meteorologische Kommission, welche den englischen Wetterdienst reorganisiert.

Herausgabe von Wetterkarten in Österreich.

1867 Wiedereinführung des britischen Sturmwarnungsdienstes (1870 68,4\% und 1872 80,5\% richtige Sturmwarnungen) wegen den Forderungen der interessierten Kreise der Schiffahrt. Dagegen wurden die täglichen Angaben des britischen Beobachtungsdienstes mit Hilfe von synoptischen Karten sorgfältig analysiert (7 Stationen erster Ordnung, 35 Stationen zweiter Ordnung, 32 Stationen dritter Ordnung) und im «Quarterly Weather Report» zur Schaffung einer wissenschaftlichen Grundlage der Wetterprognose veröffentlicht.

1869 Glaisher macht in einem Fesselballon meteorologische Beobachtungen, nachdem er schon 1862 eine Höhe von gegen 11,2 km erreicht hatte.

1871 Die Vereinigten Staaten führen Wetterkarten ein.

1872 Tägliche Wetterkarten in Großbritannien.

14.-17. August internationale meteorologische Vorversammlung in Leipzig (Provisorisches internationales Komitee).

1873 Tägliche Wetterkarten in Rußland. Der Däne HoffmeYer veröffentlicht bis 1876 tägliche Karten über den Nordatlantik.

2. bis 16. September 1873: Erster Internationaler Meteorologischer Kongreß in Wien (behandelt u. a. Vereinheitlichung und Kontrolle der Instrumente, Beobachtungstermine und Beobachtungsnetze, Berechnung der Mittelwerte, legt ein internationales Chiffresystem für Witterungstelegramme fest).

1874 Dänemark und Schweden publizieren tägliche Wetterkarten. General A. J. MYER errichtet in den Vereinigten Staaten in Verbindung mit dem in Utrecht beschlossenen simultanen Beobachtungsdienst um 0.43 Uhr ein internationales Beobachtungsnetz (108 simultane telegraphische Meldungen, 214. Postmeldungen). 
11. August bis 2. September 1874: Zweiter Kongreß für maritime Meteorologie in London.

1876 In Belgien und Deutschland erscheinen tägliche Wetterkarten.

Seit 1877 Internationalisierung des meteorologischen Dienstes (1877 Wetterkarten in Australien, internationale synoptische Karten in Österreich, 1878 in Indien, 1880 in Italien und der Schweiz, 1882 in Portugal, 1883 in Japan, 1886 in Spanien, 1890 in Holland, 1895 Kanada, 1899 Mexiko, 1902 Argentinien und Ägypten, 1906 China, 1912 Norwegen usf.)

1877-1887 Der U.S. Signal Service gibt synoptische Karten der Nordhalbkugel heraus.

1878 Einführung von Wetterprognosen in der Schweiz.

1879 Wiederaufnahme des Wettervorhersagedienstes durch den Meteorologischen Dienst Großbritanniens. Zweiter Internationaler Meteorologischer Kongreß in Rom.

1880-1910 Tägliche synoptische Karten des Nordatlantiks (gemeinsam vom Dänischen Meteorologischen Institut und der Deutschen Seewarte).

1880 Reorganisation des Schweizerischen Meteorologischen Dienstes. Ab 1881 Staatsinstitut.

1882 Errichtung einer internationalen Station auf dem Säntis (Antrag des Internationalen Meteorologischen Kongresses in Rom 1879. Mittel durch Subventionen und Sammlungen beschafft).

1882/83 Erstes internationales Polarjahr.

$1888 \quad$ Auflösung des Internationalen Meteorologischen Komitees an seiner Sitzung in Zürich.

1891 Erste Internationale Konferenz der Repräsentanten der Meteorologischen Dienste aller Länder in München.

1894/95 Erste Aufstiege mit selbstregistrierenden Instrumenten an Drachen und Seidenballons.

1896 Internationaler Wolkenatlas, 1. Ausgabe.

1901 Lehrbuch der Meteorologie von J. von HaNN (1839-1921).

1909 Erste Pilotballonaufstiege.

$1910 \quad$ Internationaler Wolkenatlas, 2. Ausgabe.

1911/12 Dynamic meteorology and hydrography von V. BJERKnes (geb. 1862). Formulierung der Polarfronttheorie der Zyclonen (Vorläufer R. Firz Roy) auf Grund einläßlicher Analysen der Wetterkarten des von der norwegischen Regierung im Interesse der Schiffahrt mit großzügigen Mitteln ausgebauten Wetterdienstes.

1914/18 Erster Weltkrieg.

nach 1918 Allgemeiner internationaler Ausbau des Wetterdienstes. Einführung der Funktelegraphie. Zunehmende Verwertung von aerologischen Aufstiegen und Radiosonden.

1928 Eiskeimtheorie des Regens von BERGERON.

1932/33 Zweites internationales Polarjahr.

1939 Sekretariat der Internationalen Meteorologischen Organisation in Lausanne.

1939/45 Zweiter Weltkrieg.

1940 Theorie der Gewitterelektrizität von FindeISEN.

nach 1945 Internationaler Ausbau des Flugwetterdienstes. Installation von Wetterschiffen mit Funkstationen auf dem Weltmeer, speziell dem Nordatlantik. 


\section{B IBLI O G RAPH IE}

P.W.Sidler, Zur Entwicklungsgeschichte der modernen Meteorologie. Jahresberichte der Lehr- und Erziehungsanstalt Einsiedeln 1876/77 und 1877/78.

G.Hellmann, Beiträge zur Geschichte der Meteorologie. Berlin 1914, 1917, 1922.

Dr.A.Defant, Wetter und Wettervorhersage (Synoptische Meteorologie). Leipzig/Wien 1926.

Sir Napier Shaw, Manuel of Meteorology. Volume I: Meteorology in History. Cambridge 1932.

J. von HANN und R.Süring, Lehrbuch der Meteorologie. 3. Auflage, 1939.

Cнвомоw und SwовоDA, Einführung in die synoptische Wetteranalyse. 1940.

R. Billwiller, Meteorologie, in der Festschrift zur 200-Jahr-Feier der Naturforschenden Gesellschaft in Zürich, 1946.

H. FICKER, Synoptisch-meteorologische Forschung in der Gegenwart, in Experientia IV, 139 und 165 (1948).

Zahlreiche kleinere Studien von G. HellmanN, H. Wild u. a.

\section{Anhang II. Chronologische Übersicht der Entwicklung der Nachrichtentechnik und Nachrichtenübermittlung}

1792 Erfindung des optischen Telegraphen (Gebrüder Chappe).

1794 Linie Paris-Lille mit zwanzig Stationen (Übermittlung der Nachricht der Rückeroberung der Stadt Condé an den Konvent in zwei Minuten).

1794/1833 Ausbau des Systems der optischen Telegraphie für staatliche Zwecke (1797 Paris-Straßburg, 1798 Paris-Brest, 1805 Paris-Lyon, 1816 Paris-Calais, 1821 Lyon-Toulon, 1823 Paris-Bordeaux, 1833 Berlin-Koblenz-Köln-Trier usw.).

nach 1794. Zahlreiche Versuche, elektrische Telegraphen zu erfinden (Funkentelegraphen Reozen 1794, Salvos 1798, Ronalds 1810; elektrolytische Telegraphen Salva 1805, Soemmering 1809; elektromagnetische Telegraphen Ampère 1821, Cook und Wheatstone 1829; Zeigertelegraph Wheatstone 1839 u.a. mehr).

1831/32 Faradays Versuche über die Induktion. Morse wird dadurch auf der Rückfahrt von Europa nach Amerika zur Konstruktion eines elektromagnetischen Schreibtelegraphen angeregt.

$1835 \quad$ Erstes Modell des Morseapparates.

1836 Doppelmodell des Morsetelegraphen.

7. April 1838: Morse meldet seinen Apparat dem Patentamt an.

$1839 \quad$ Erste Telegraphenlinie Washington-Baltimore.

1844 Erste amerikanische Telegraphengesellschaft (1851 bestehen bereits fünfzig amerikanische Gesellschaften).

1845 Einführung von Relaisstationen durch Morse und Henri. Versuchsstrecke Paris-Rouen.

1846 Das Einleitersystem mit der Erde als Rückleitung wird durch das Zweileitersystem ersetzt.

1853 Das Duplexsystem erlaubt das gleichzeitige Telegraphieren in beiden Richtungen. 
1858 Das erste Überseekabel von Irland nach Neufundland bricht nach drei Monaten.

1865 Gründung des Internationalen Telegraphenvereins in Paris durch zwanzig europäische Staaten unter Fusion des 1850 gegründeten Deutsch-Österreichischen Telegraphenvereins mit dem 1852 gegründeten Westeuropäischen Telegraphenverein.

1866 Erste erfolgreiche Kabelverbindung von Europa nach Amerika.

1876 Prof. Alex. Graham Bell erfindet in Boston das Telephon (erstes Gespräch am 10. März 1876).

1878 Ausstellung des Telephons an der Pariser Weltausstellung. Rasche Ausbreitung des Telephons in den Vereinigten Staaten und Europa.

1880 Erste schweizerische Telephonzentrale in Zürich.

1886 Verstaatlichung des Telephonwesens in der Schweiz.

1887 H. Hertz (1857-1894) erzeugt elektromagnetische Wellen.

1890 Der Franzose Branly erfindet den Kohärer.

1895 Der Russe Popoff erfindet die Antenne.

1896 Der Italiener MARconI erfindet unter Verwendung von Antenne, Kohärer und anderen physikalischen Apparaten die drahtlose Telegraphie.

1898 Prof. Braun in Straßburg konstruiert den geschlossenen Schwingungskreis.

1903 Gründung der Gesellschaft für drahtlose Telegraphie (Telefunkensystem).

1906 Erfindung der Elektronenröhre durch LIEBEN-REIss und Forest.

\section{Buchbesprechungen}

1. Cyril Elgood, A Medical History of Persia and the Eastern Caliphate from the Earliest Times until the Year A.D. 1932. With 6 Plates. XII 617 Seiten. University Press, Cambridge, 1951. 50sh.

Sir Cyril Elgoon kommt das große Verdienst zu, die Medizingeschichte Persiens von den ältesten Zeiten, über die Avesta-Medizin (Vendidad usw.), den Beginn der eigentlich geschichtlichen Zeit der Achaemenidenkönige (etwa von 650 v. Chr. an) mit Darius und dem griechischen Leibarzt Demokedes, mit Artaxerxes I. und dem griech. Leibarzt Ktesias, dessen Commentarii Medici leider verloren sind, über die Alexanderzeit, über die Blüteperiode der arabischen Ärzte bis in die Gegenwart zu führen. ElGood ist als britischer Gesandtschaftsarzt in Teheran geradezu prädestiniert, diese Geschichte aus genauester Lokal- und vielseitiger Quellenkenntnis zu schreiben.

Von besonderem Interesse für den Medizinhistoriker ist neben der islamitischen Periode die noch wenig erhellte Periode vom Tode Alexanders des Grossen bis zur Gründung des Islams: die Zeit der zoroastrischen Reform unter ArDESHIR zu Beginn des 3. Jahrhunderts n. Chr. Sein Nachfolger Shapur I. († 271) sammelte in großem Maßstab die Pahlevitexte der avestischen Zeit. In Jundi Shapur gründete Shapur II. jene berühmte Universität und Medizinschule, an welcher seit 